\title{
Dietary Geraniol by Oral or Enema Administration Strongly Reduces Dysbiosis and Systemic Inflammation in Dextran Sulfate Sodium-Treated Mice
}

\begin{abstract}
Luigia De Fazio ${ }^{1}$, Enzo Spisni ${ }^{1 *}$, Elena Cavazza ${ }^{1}$, Antonio Strillacci ${ }^{1}$, Marco Candela ${ }^{2}$, Manuela Centanni ${ }^{2}$, Chiara Ricci ${ }^{3}$, Fernando Rizzello ${ }^{4}$, Massimo Campieri ${ }^{4}$ and Maria C. Valerii ${ }^{1}$

${ }^{1}$ Biology Unit, Department of Biological, Geological and Environmental Sciences, University of Bologna, Bologna, Italy, ${ }^{2}$ Department of Pharmacy and Biotechnology, University of Bologna, Bologna, Italy, ${ }^{3}$ Department of Clinical and Experimental Sciences, University of Brescia, Brescia, Italy, ${ }^{4}$ Department of Medical and Surgical Sciences, University of Bologna, Bologna, Italy
\end{abstract}

(Trans)-3,7-Dimethyl-2,6-octadien-1-ol, commonly called geraniol (Ge-OH), is an acyclic monoterpene alcohol with well-known anti-inflammatory, antitumoral, and antimicrobial properties. It is widely used as a preservative in the food industry and as an antimicrobial agent in animal farming. The present study investigated the role of $\mathrm{Ge}-\mathrm{OH}$ as an anti-inflammatory and anti-dysbiotic agent in the dextran sulfate sodium (DSS)-induced colitis mouse model. Ge-OH was orally administered to C57BL/6 mice at daily doses of 30 and $120 \mathrm{mg} \mathrm{kg}^{(-1)}$ body weight, starting 6 days before DSS treatment and ending the day after DSS removal. Furthermore, $\mathrm{Ge}-\mathrm{OH} 120 \mathrm{mg} \mathrm{kg}^{(-1)}$ dose body weight was administered via enema during the acute phase of colitis to facilitate its on-site action. The results show that orally or enema-administered $\mathrm{Ge}-\mathrm{OH}$ is a powerful antimicrobial agent able to prevent colitis-associated dysbiosis and decrease the inflammatory systemic profile of colitic mice. As a whole, Ge-OH strongly improved the clinical signs of colitis and significantly reduced cyclooxygenase-2 (COX-2) expression in colonocytes and in the gut wall. Ge-OH could be a powerful drug for the treatment of intestinal inflammation and dysbiosis.

Keywords: geraniol, inflammatory bowel disease (IBD), dextran sulfate sodium (DSS)-induced colitis,
cyclooxygenase-2 (COX-2), inflammation, dysbiosis

Received: 30 November 2015 Accepted: 11 February 2016 Published: 03 March 2016

Citation: De Fazio L, Spisni E, Cavazza E, Strillacci A, Candela M, Centanni M Ricci C, Rizzello F, Campieri $M$ and Valerii MC (2016) Dietary Geraniol by Oral or Enema Administration Strongly

Reduces Dysbiosis and Systemic Inflammation in Dextran Sulfate Sodium-Treated Mice.

Front. Pharmacol. 7:38. doi: 10.3389/fphar.2016.00038

\section{INTRODUCTION}

More than $90 \%$ of the 100 trillion cells in the human body are microbes, most of which reside in the digestive tract and are collectively known as the intestinal microbiota (Yaung et al., 2014). The bacterial flora is extremely dense and diverse and shapes fundamental physiological processes such as digestion and the development of gut-associated lymphoid tissues and systemic immunity. The intestinal microbiota plays a crucial role in maintaining colonic homeostasis, while microbial dysbiosis can contribute to a wide spectrum of disease (Kamada et al., 2013).

Abbreviations: Ge-OH, geraniol; DSS, Dextran sulfate sodium; IBD, Inflammatory bowel disease; COX-2, Cyclooxygenase-2; CRC, Colorectal cancer. 
Inflammatory bowel disease (IBD), which includes Crohn's disease (CD), and ulcerative colitis (UC), is a chronic inflammatory disorder of the intestinal tract associated with abdominal pain, intestinal bleeding, weight loss, and diarrhea (Koloski et al., 2008). The etiology of IBD is unknown but the one dominant hypothesis is that the inflammation results from altered or pathogenic microbiota in a genetically susceptible host. A growing body of literature implicates the abnormal overgrowth or dominance of particular bacterial species in the pathogenesis of IBD. Notably, mouse model studies of IBD have shown protection against the development of IBD in a germ-free environment, corroborating the role of gut flora in the pathogenesis of this spectrum of illnesses (Missaghi et al., 2014).

As in humans, the two most abundant bacterial phyla in C57BL6/J mice are the Firmicutes (60-80\% of sequences) and the Bacteroidetes (20-40\%). Few bacteria are present in the mouse gut soon after birth. The neonate is inoculated with microorganisms by the mother and the environment and the microbiota is fully established when the mouse reaches adulthood at around 8 weeks, even if it is still susceptible to changes in composition (Laukens et al., 2015). In healthy adults, diet changes remain the major player in microbiota dynamics.

Essential oil mixtures have been shown to play a significant role in the modulation of animal gut microbiota (OviedoRondón et al., 2006) but their mechanism(s) of action remain incompletely understood (Thompson et al., 2013).

Essential oils (EO) are volatile natural complex compounds characterized by a strong odor and synthesized by aromatic plants as secondary metabolites (Bakkali et al., 2008). They are highly complex natural mixtures which may contain up to 60 components at widely varying concentrations. In nature, EO play important roles in the protection of plants acting as antibacterial, antiviral, antifungal, and insecticidal agents (Bakkali et al., 2008; Fang et al., 2010). Recently, EO have been used in animal feed to treat infections, manipulate gut fermentation, and improve productivity (Wallace et al., 2010).

Geraniol $(\mathrm{Ge}-\mathrm{OH})$ is a naturally acyclic monoterpene component of EO extracted from lemongrass, rose, and other aromatic plants. Several studies on the biological activities of Ge$\mathrm{OH}$ have shown it to be a highly active antitumoral, antimicrobial compound, with antioxidant and anti-inflammatory properties (Ahmad et al., 2011; Thapa et al., 2012; Khan et al., 2013).

Ge-OH's antimicrobial activities do not seem to have specific cellular targets. Like other $\mathrm{EO}, \mathrm{Ge}-\mathrm{OH}$ is a hydrophobic compound able to bind to the bacterial wall modifying its dynamic organization, with a consequent loss of ions and ATP depletion (Di Pasqua et al., 2006; Turina et al., 2006). In addition to bacterial growth inhibition, especially effective on Gram-positive bacteria (Thapa et al., 2012), Ge-OH also damages bacterial proteins, and lipids (Burt, 2004; Oussalah et al., 2007). Ge-OH effectively modulates the drug resistance of several Gram-negative bacterial species such as E. aerogenes, $E$. coli, and $P$. aeruginosa by restoring drug susceptibility in strains overexpressing efflux pumps (Solórzano-Santos and MirandaNovales, 2012). It is important to emphasize that human pathogenic bacteria are more sensitive to $\mathrm{Ge}-\mathrm{OH}$ than are commensal species even if the nature of this selectivity remains unsettled (Singh et al., 2012).

$\mathrm{Ge}-\mathrm{OH}$ has antioxidant activities in eukaryotic cells (Khan et al., 2013). By reducing oxidative stress, Ge-OH may prevent drug-induced mitochondrial dysfunction in hepatocytes (Singh et al., 2012). In vivo, it proved able to enhance neurodegeneration in a mice model of Parkinson's disease (Rekha et al., 2013).

In vitro and in vivo, Ge-OH inhibits the expression of cyclooxygenase-2 (COX-2; Chaudhary et al., 2013), a key enzyme in inflammation (Strillacci et al., 2010). The anti-inflammatory properties of $\mathrm{Ge}-\mathrm{OH}$ have been assessed on different animal models and in this context it has been shown that its molecular target is not only COX-2 but also NF-kB (Marcuzzi et al., 2011; Khan et al., 2013; Medicherla et al., 2015).

Considering all its activities, $\mathrm{Ge}-\mathrm{OH}$ seems to be an excellent candidate for the treatment of gut and systemic inflammations and for the control of gut dysbiosis. Medicherla et al. (2015) have already proved that $\mathrm{Ge}-\mathrm{OH}$ effectively modulates experimental colitis, but the possibility of using this molecule as a therapeutic agent has yet to be demonstrated. Their study did not consider the chemical characteristics of $\mathrm{Ge}-\mathrm{OH}$ that require specific formulations to be administered. They administered $\mathrm{Ge}-\mathrm{OH}$ orally diluted in saline, forgetting that $\mathrm{Ge}-\mathrm{OH}$ is insoluble in aqueous solutions in which it rapidly tends to separate from the water. Moreover, once separated from water, $\mathrm{Ge}-\mathrm{OH}$ reaches high concentrations at which it could irritate the gut mucosa. Since, this substance rapidly crosses enterocyte monolayers (Heinlein et al., 2014), its site of action and its impact on the microbiota should also be evaluated. To determine whether $\mathrm{Ge}-\mathrm{OH}$ could become a therapeutic option in humans, we administered $\mathrm{Ge}-\mathrm{OH}$ in appropriate oral or enema formulations to dysbiotic mice and compared its effects with one of the standard therapies currently used to manage gut inflammation in IBD patients.

\section{MATERIALS AND METHODS Ge-OH Oral Formulation}

$\mathrm{Ge}-\mathrm{OH}$ oral formulation was optimized for the administration route chosen and for a possible transition to use in humans as it has a strong smell and very unpleasant taste. In addition, Ge$\mathrm{OH}$ is completely water insoluble and could irritate the mucosae if administered pure. The oral formulation was then optimized for a slow release of $\mathrm{Ge}-\mathrm{OH}$ using a patented soy lecithin incapsulation. Natural Ge-OH (analytical grade, $>98 \%$ pure) and soy lecithin were purchased from Prodasynth (Grasse, France). All the other reagents were purchased from SIGMA-Aldrich (St Louis, MO, USA). The stable suspension was prepared by Cedax Srl (Forli, Italy) by adding Ge-OH $\left(\rho=0.899 \mathrm{~g} / \mathrm{cm}^{3} ; 17 \%\right.$ by weight) to a solution containing sucrose (16\%), deionized water $(22 \%)$ and soy lecithin (25\%), and ethanol $(20 \%)$ as preservative (patent PCT WO 201 1/128597). The suspension was stored at $4{ }^{\circ} \mathrm{C}$ and administered by oral gavage to $\mathrm{Ge}-\mathrm{OH}-$ treated mice $(4,5$, or $18 \mu \mathrm{l}$ of $\mathrm{Ge}-\mathrm{OH}$ suspension brought to the final volume of $100 \mu \mathrm{l}$ with $\mathrm{Ge}-\mathrm{OH}$-free suspension). A $\mathrm{Ge}-\mathrm{OH}$-free suspension containing sucrose $(16 \%)$, soya lecithin (25\%), and ethanol (20\%) was administered to the control group. 


\section{Ge-OH Enema Formulation}

The $\mathrm{Ge}-\mathrm{OH}$ formulation for enema administration was prepared using glycerin to increase the viscosity of the solution and thereby facilitate both intracolonic injection and colonic retention. Enema $\mathrm{Ge}-\mathrm{OH}$ solution was prepared as follows: natural $\mathrm{Ge}-\mathrm{OH}$ $(4 \% \mathrm{v} / \mathrm{v})$ was added to a solution containing PBS and glycerol $(30 \% \mathrm{v} / \mathrm{v})$. An amount of solution corresponding to $120 \mathrm{mg} \mathrm{kg}^{(-1)}$ (body weight, die) was freshly prepared and administered by enema during the acute phase of colitis. A control solution mixed as previously described but without $\mathrm{Ge}-\mathrm{OH}$ was also prepared and administered to the enema control group. Enema treatments were administered via a $16 \mathrm{G}$ venous catheter (diameter $2 \mathrm{~mm}$, length $48 \mathrm{~mm}$; BD Bioscience, Buccinasco, Italy) advanced through the rectum into the colon until the tip was $10 \mathrm{~mm}$ proximal to the anus. A venous catheter was applied to a $1 \mathrm{ml}$ syringe and the suspension was gently injected into the rectum. Animals were sedated using tiletamine $10 \mathrm{mg} \mathrm{kg}^{(-1)}$ plus xylazine $2.5 \mathrm{mg} \mathrm{kg}^{(-1)}$ during enema administration.

\section{Hydrocortisone Enema Treatment}

Enema administrations were prepared as follows: hydrocortisone $(0.08 \% \mathrm{w} / \mathrm{v}$, Sigma) was added to a solution containing PBS and glycerol $(30 \% \mathrm{v} / \mathrm{v})$. An amount of solution corresponding to $2.5 \mathrm{mg} \mathrm{kg}^{(-1)}$ (body weight, die) was freshly prepared and administered by enema during the acute phase of colitis. Enema treatments were administered via a $16 \mathrm{G}$ venous catheter as previously described.

\section{Animal Treatment}

Sixty-four eight-week-old male C57BL/6 mice were purchased from Charles River Laboratories (Lecco, Italy). Animals were housed in collective cages with a controlled environment containing two mice each, at $22 \pm 2{ }^{\circ} \mathrm{C}$ and $50 \%$ humidity, under a 12-h light/dark cycle. Mice were allowed to acclimate to these conditions for at least 7 days before inclusion in experiments and had free access to food and water throughout the study.

Mice were randomized into eight experimental groups: the first (I) group called CTRL $(n=8)$ received only tap water for 37 days (1-37). Group II called SoySusp-or received only tap water for 37 days (1-37) and mice were treated with oral $\mathrm{Ge}-\mathrm{OH}$-free suspension for 17 days (days 8-24) to analyze possible modifications of the microbiota induced by the soy lecithin suspension in healthy mice. All the groups III-VIII received tap water for 16 days (1-16), oral administration of $1.5 \%$ $(\mathrm{w} / \mathrm{v})$ dextran sulfate sodium (DSS for colitis, TdB Consultancy, Sweden) for 7 days in tap water (days 17-23), and tap water for 14 days (25-37). DSS was freshly prepared every 7 days and the average amount of DSS taken was recorded daily. In addition to DSS, group III, called DSS $(n=8)$, received the control oral Ge-OH-free suspension for 17 days (days 8-24), while group IV, called DSS + Ger30 or $(n=8)$, received Ge-OH orally [30 mg $\mathrm{kg}^{(-1)}$ ] for 17 days (days 8-24). Group V, called DSS+Ger120 or $(n=8)$, received $\mathrm{Ge}-\mathrm{OH}\left[120 \mathrm{mg} \mathrm{kg}^{(-1)}\right]$ for 17 days (days 8$24)$, and group VI $(n=8)$, called DSS + Ger120 En, received four enema administrations of $\mathrm{Ge}-\mathrm{OH}$ on experimental days 19, 21, 23 , and 25. Group VII $(n=8)$, called DSS+Susp enema, received four enema administrations of glycerol-PBS suspension on days $19,21,23$, and 25 .

The last group, VIII, called DSS+hydrocortisone enema, received four enema administrations of glycerol-PBShydrocortisone on days 19,21,23, and 25. This group was used as a model to understand how colitis is clinically modulated by a powerful drug. Hydrocortisone was administered by enema at doses of $2.5 \mathrm{mg} \mathrm{kg}^{(-1)}$ body weight.

The experimental design is schematized in Figure 1. The experiments were carried out in accordance European and Italian guidelines. They were approved by the Institutional Ethical Review Board of the University of Bologna and by the Italian Ministry for Research and were repeated twice.

\section{Disease Activity Index (DAI)}

DAI was calculated by the combined score of weight loss, stool consistency and bleeding, as detailed in Table 1. All parameters were scored from day 1 to day 37.

\section{Histological Evaluation of Colitis}

Mice ( $n=2$ for each experimental group) were anesthetized using Zoletil-100 [10 mg kg(-1); Virbac, Carros, France], and
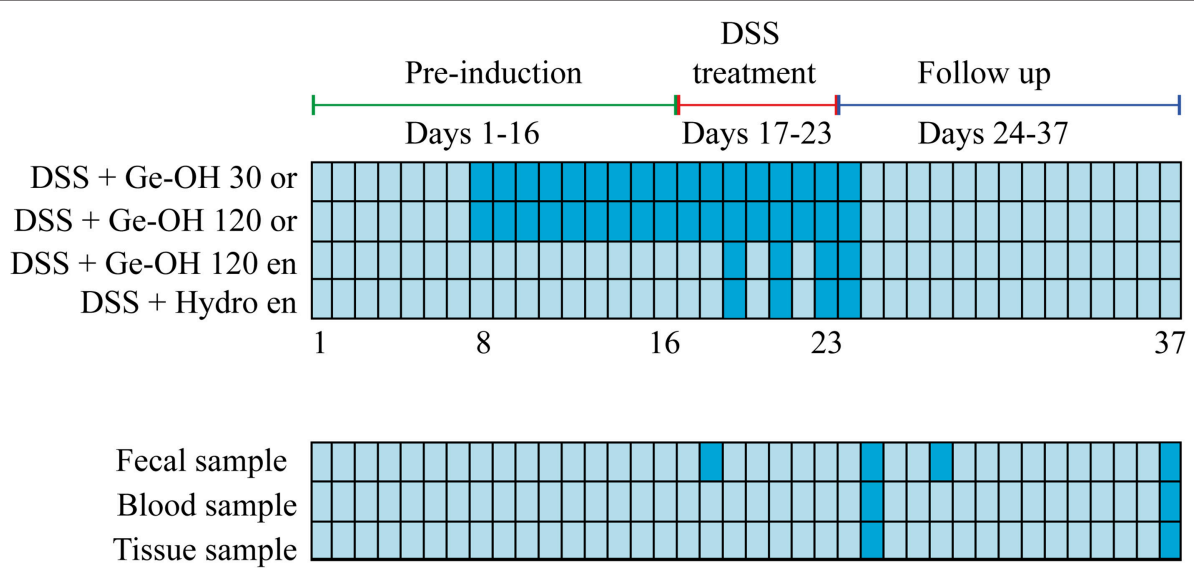

FIGURE 1 | Experimental design of the study. Animal treatment and the collection of feces, blood, and tissue are indicated (dark blue) in the grid. 
TABLE 1 | Disease activity index (DAI) score parameters.

\begin{tabular}{lll}
\hline Stool consistency & Bleeding & Weight loss \\
\hline $0=$ Formed & $0=$ Normal color stool & $0=$ No weight loss \\
$1=$ Mild-soft & $1=$ Brown color & $1=5-10 \%$ weight loss \\
$2=$ Very soft & $2=$ Reddish color & $2=11-15 \%$ weight loss \\
$3=$ Watery stool & $3=$ Bloody stool & $3=16-20 \%$ weight loss \\
& & $4=>20 \%$ weight loss
\end{tabular}

Xilor [2.5 $\mathrm{mg} \mathrm{kg}^{(-1)}$; Bio98, Milan, Italy] by intramuscular injection and sacrificed by cervical dislocation on day 25 (2 days after the end of DSS treatment, when the maximum DAI score was reached), and day 37, at the end of weight recovery. The colon was excised, rinsed with saline solution, fixed in $4 \%$ formalin and embedded in paraffin. Four micrometer sections were stained with hematoxylin-eosin and observed for histological assessment of epithelial damage by a pathologist in a blinded manner.

\section{Determination of Plasma Cytokine Levels}

Blood samples $(200 \mu \mathrm{l})$ were taken from the tail vein on days 25 and 37 and collected in Eppendorf tubes. Blood was centrifuged at $1000 \mathrm{rpm}$ for $10 \mathrm{~min}$, and plasma was collected and stored at $-80^{\circ} \mathrm{C}$ until BioPlex analysis. Cytokine levels were determined using a multiplexed mouse bead immunoassay kit (Bio-Rad, CA, USA). The six-plex assays (IL-1 $\beta$, IL-6, IL-10, IL$17 \mathrm{~A}, \mathrm{IFN} \gamma, \mathrm{TNF} \alpha$ ) were performed in 96-well plates following the manufacturer's instructions. Microsphere magnetic beads coated with monoclonal antibodies against the different target analytes were added to the wells. After $30 \mathrm{~min}$ incubation, the wells were washed and biotinylated secondary antibodies were added. After incubation for $30 \mathrm{~min}$, beads were washed and then incubated for $10 \mathrm{~min}$ with streptavidin-PE conjugated to the fluorescent protein, phycoerythrin (streptavidin/phycoerythrin). After washing, the beads (a minimum of 100 per analyte) were analyzed in the BioPlex 200 instrument (BioRad). Sample concentrations were estimated from the standard curve using a fifth-order polynomial equation and expressed as $\mathrm{pg} / \mathrm{ml}$ after adjusting for the dilution factor (Bio-Plex Manager software 5.0). The sensitivities of the assay were $3.14 \mathrm{pg} / \mathrm{ml}$ (IL-1 $\beta), 1.34 \mathrm{pg} / \mathrm{ml}$ (IL-6), $1.38 \mathrm{pg} / \mathrm{ml}$ (IL-10), $2.38 \mathrm{pg} / \mathrm{ml}$ (IL-17), $1.38 \mathrm{pg} / \mathrm{ml}$ (IFN $\gamma$ ), and $2.73 \mathrm{pg} / \mathrm{ml}(\mathrm{TNF} \alpha)$. Samples below the detection limit of the assay were recorded as zero. The intra-assay CV was $<14 \%$.

\section{Characterization of the Intestinal Microbiota by HTF-Microbi.Array}

The intestinal mice microbiota was characterized using the fully validated diphylogenetic DNA microarray platform HTFMicrobi.Array. Targeting 33 phylogenetically related groups, this LDR-based universal array covers up to $95 \%$ of the mammalian gut microbiota. Gut microbiota analysis was performed on days 18, 25, 29, and 38. Total DNA from fecal material was extracted using the QIAamp DNA Stool Mini Kit (Qiagen) according to the modified protocol previously reported (Candela et al., 2010, 2012). Final DNA concentration was determined using NanoDrop ND-1000 (NanoDrop Technologies). A nearly full-length portion of $16 \mathrm{~S}$ rDNA gene was amplified using universal forward primer $27 \mathrm{~F}$ and reverse primer $1492 \mathrm{R}$, according to the protocol previously described (Castiglioni et al., 2004) PCR amplifications were performed in a Biometra Thermal Cycler T Gradient (Biometra, Göttingen, Germany). PCR products were purified using the High Pure PCR Cleanup Microkit (Roche, Mannheim, Germany), eluted in $30 \mu \mathrm{l}$ of sterile water and quantified with NanoDrop ND-1000. Slide chemical treatment, array production, LDR protocol, and hybridization conditions were performed as previously reported (Candela et al., 2012). Briefly, LDR reactions were carried out in a final volume of $20 \mu \mathrm{l}$ containing $500 \mathrm{fmol}$ of each LDR-UA HTF-Microbi.Array probe, $50 \mathrm{fmol}$ of PCR product, and 25 fmol of the synthetic template (5'-AGCCGCGAACACCACGAT CGACCGGCGCGCGCAGCTGCAGCTTGCTCATG-3'). LDR products were hybridized on universal arrays, setting the probe annealing temperature at $60^{\circ} \mathrm{C}$. All arrays were scanned and processed according to the protocol and parameters already described. Fluorescence intensities were normalized on the basis of the synthetic ligation control signal. The relative abundance of each bacterial group was obtained by calculating the relative fluorescence contribution of the corresponding HTFMicrobi.Array probe as a percentage of the total fluorescence.

\section{RNA Extraction and Real-Time PCR}

Colon specimens were collected immediately after sacrifice and total RNA was extracted using Trizol $^{\circledR}$ reagent (Life Technologies, CA, USA) according to the manufacturer's instructions. Extracted RNA samples were treated with DNase I to remove any genomic DNA contamination using DNA-free kit (Ambion, USA) and reverse-transcripted using RevertAid $^{\mathrm{TM}}$ First Strand cDNA Synthesis Kit (Fermentas, Canada). COX-2 and $\beta$-actin mRNAs were reverse-transcribed using random hexamer primers (Fermentas, Canada). COX-2 and $\beta$-actin mRNA levels were analyzed by real-time PCR using SYBR ${ }^{\circledR}$ Select Master Mix (Life Technologies, CA, USA) and StepOnePlusTM system (Applied Biosystems, CA, USA) according to the manufacturers' instructions. The melting curve data were collected to check PCR specificity. Each cDNA sample was analyzed in triplicate. COX-2 mRNA levels were normalized against $\beta$-actin mRNA and relative expressions were calculated using the $2-2 \Delta \mathrm{Ct}$ formula. COX-2 primer pair: $5^{\prime}-\mathrm{TTC}$ TCT ACA ACA ACT CCA TCC TC $-3^{\prime}$ and $5^{\prime}-$ GCA GCC ATT TCC TTC TCT CC - $3^{\prime}$ (247 bp product); $\beta$-actin primer pair: $5^{\prime}$ - ACC AAC TGG GAC GAC ATG GAG $-3^{\prime}$ and $5^{\prime}$ - GTG GTG GTG AAG CTG TAG CC -3' (380 bp product).

\section{Data Analysis}

Statistical analysis was carried out using GraphPad Prism 6 (GraphPad Software Inc., San Diego, CA, USA). Data are expressed as mean \pm SEM of at least three independent determinations. Student's $t$-test, analysis of variance (one-way ANOVA) followed by Bonferroni's post-hoc test for multiple comparison were used to assess the statistical significance of the differences. Differences were considered statistically significant at $P<0.05$. Euclidean distance of HTF-Microbi.Array relative abundance profiles were used to perform PCoA and analysis 
was accomplished using the $\mathrm{R}$ packages Made4, Vegan, and Stats (www.cran.org).

\section{RESULTS}

\section{Clinical Colitis Activity}

The effect of DSS and DSS-Ge-OH treatments was evaluated considering the DAI calculated as the sum of weight loss, stool consistency, and blending scores (Table 1). All DSS-treated mice started to show mild clinical signs of disease 2 days before the end of the 1.5\% DSS treatment (day 21) due to the simultaneous increase in stool consistency index and bleeding index (maximum DAI score $=2.3$ ). The most evident clinical signs of each group were recorded between days 25 and 27 (Figure 2) with a maximum DAI score of 9.1 for the DSS group and with severe weight loss that peaked between days 25 and 28 (Figure 2A). Ge-OH at $30 \mathrm{mg} \mathrm{kg}^{(-1)}$ reduced the DAI score of colitis during the acute phase but did not affect this index during the recovery phase (Figure $2 \mathbf{B}$ ). At this $\mathrm{Ge}-\mathrm{OH}$ dose, the DAI score maintained the same trend observed in DSStreated mice. At the higher oral dose, $\mathrm{Ge}-\mathrm{OH}$ reduced the DAI score for almost the entire duration of colitis and especially during the recovery phase. Statistical analysis of data in Figure 2B are provided in Supplementary Table 1. These positive Ge-OH effects were further enhanced when colitic mice were treated with enema-administered $\mathrm{Ge}-\mathrm{OH}$, resulting in a very low weight loss and a strongly reduced DAI score for the whole duration of colitis.

\section{Inflammatory Cytokine Profile of Colitis}

Plasma levels of IL-1 $\beta$, IL-6, IL-10, IL-17, TNF $\alpha$, and IFN $\gamma$ were detected in blood samples from all experimental mice group at two different time points, one corresponding to the acute phase of colitis (day 25), and one at the end of the recovery phase (day 37). DSS treatment significantly increased $(P<0.05)$ all the cytokines measured, both at day 25 and day 37 (Figure 3). At day 25, oral administration of $\mathrm{Ge}-\mathrm{OH}$ at the lower dose of $30 \mathrm{mg} / \mathrm{mg} \mathrm{kg}(-1)$ did not modify the inflammatory profile of DSS-treated mice. Oral administration of the higher $\mathrm{Ge}-\mathrm{OH}$ dose of $120 \mathrm{mg} \mathrm{kg}^{(-1)}$ and $\mathrm{Ge}-\mathrm{OH} 120 \mathrm{mg} \mathrm{kg}^{(-1)}$ enema administration significantly decreased IL-10, IL-17, TNF $\alpha$, and IFN $\gamma(P<0.05)$, but neither IL-1 $\beta$ nor IL- 6 . At day 37 when colitis tended to become chronic, Ge-OH-treated mice showed a better inflammatory profile than DSS-treated mice. In particular, the lower dose of oral Ge-OH significantly reduced all the measured cytokines $(P<0.05)$. The higher oral dose and enema administration of Ge-OH significantly decreased IL- $1 \beta$, IL-17, IFN $\gamma$, and TNF $\alpha(P<0.05)$ but neither IL-6 nor IL-10.

\section{Histological Evaluation of Colitis}

Histological evaluation of the colon was made from the colocecal junction to the anus. Overall, the tissue damage tended to be limited to the terminal colon and rectum regions, and can be classified as moderate colitis (Figure 4). At day 25 (Figures 4A-C), the colon mucosa in the DSS-treated mice showed a diffuse loss of goblet cells, focal crypt abscesses, diffuse hyperemia, moderate cellular infiltration in the mucosa, and focal epithelial erosions. Diffuse hyperemia, mild loss of goblet cells, mild cellular infiltration but no crypt abscesses, or epithelial erosions were also present in the mucosa of oral $\mathrm{Ge}-\mathrm{OH}$-treated mice at both doses administered [see Supplementary Figure 1 for $\left.\mathrm{Ge}-\mathrm{OH} 30 \mathrm{mg} \mathrm{kg}^{(-1)}\right]$. The colon in the Ge-OH enema-treated mice was characterized by a lower mucosa distortion (elongation) and showed moderate loss of epithelium, and low leukocyte infiltrations.

After weight recovery (day 37), the colon mucosa in the DSS-treated mice showed a diffuse loss of goblet cells, focal crypt abscesses, diffuse hyperemia, and mild cellular infiltration (Figure 4D), while the mucosa of oral $\mathrm{Ge}-\mathrm{OH}$-treated mice presented diffuse hyperemia but a milder loss of goblet cells, a milder cellular infiltration, and no crypt abscesses at with dose administered (Figures 4E,F). Colon mucosa in the enema Ge-OH-treated mice showed a normal architecture similar to that of healthy controls. In conclusion, histological and clinical improvements were evident in the $\mathrm{Ge}-\mathrm{OH}$-treated mice and particularly in the enema-treated animals.

\section{Ge-OH-Induced Microbiota Modifications}

Since the Ge-OH-free suspension itself did not induce microbiota alterations, we investigated the impact of $\mathrm{Ge}-\mathrm{OH}$ treatment on DSS-induced microbiota dysbiosis in mice. Mice stools were collected on days 18, 25, 29, and 37. Figure 5 shows the phylogenetic structure of the intestinal microbiota characterized using the HTF-Microbi.Array universal platform. DSS treatment prompted profound, progressive, and transient changes in mice microbiota composition, compared to colitis-negative controls (group I), defining a peculiar microbiota trajectory during the induced colitis. In particular, on day 18, after 1 day of DSS treatment, the overall microbiota structure of DSS mice still resembled that of control mice. At day 25, after seven days of DSS, we observed a global temporary restructuring of the intestinal microbiota composition. At day 29 a transitory reduction of Bacteroidetes associated with an increase in Firmicutes was recorded. However, on day 37, DSS-treated mice recovered a microbiota structure similar to that of healthy controls.

While oral $\mathrm{Ge}-\mathrm{OH}$ treatment at $30 \mathrm{mg} \mathrm{kg}{ }^{(-1)}$ exerted only a mild impact on the temporal dynamics of DSS-induced microbiota dysbiosis, oral and enema treatment at a dose of $120 \mathrm{mg} \mathrm{kg}^{(-1)}$ resulted in considerable protection against the transient DSS-dependent reduction of Bacteroidetes, favoring a faster recovery of a community profile similar to that of healthy controls. In particular, on day $25, \mathrm{Ge}-\mathrm{OH}$ at $120 \mathrm{mg} \mathrm{kg}^{(-1)}$ (both enema and orally administered) triggered a Lactobacillaceae increase that reached a relative abundance of 11.2 and 9.7\% respectively, notably higher than the corresponding value in control mice. This Ge-OH-dependent high relative abundance of Lactobacillaceae was maintained until day 29 after which Ge$\mathrm{OH}$-treated mice permanently recovered from the DSS-induced reduction of Bacteroidetes 8 days earlier with respect to the corresponding DSS-treated mice. These effects are certainly related to the antibacterial action of $\mathrm{Ge}-\mathrm{OH}$, evidenced by its low minimal inhibitory concentration (MIC) on model bacteria species (see Supplementary Table 2). Differently from what observed in DSS treated mice, in healthy mice Ge-OH treatment, 


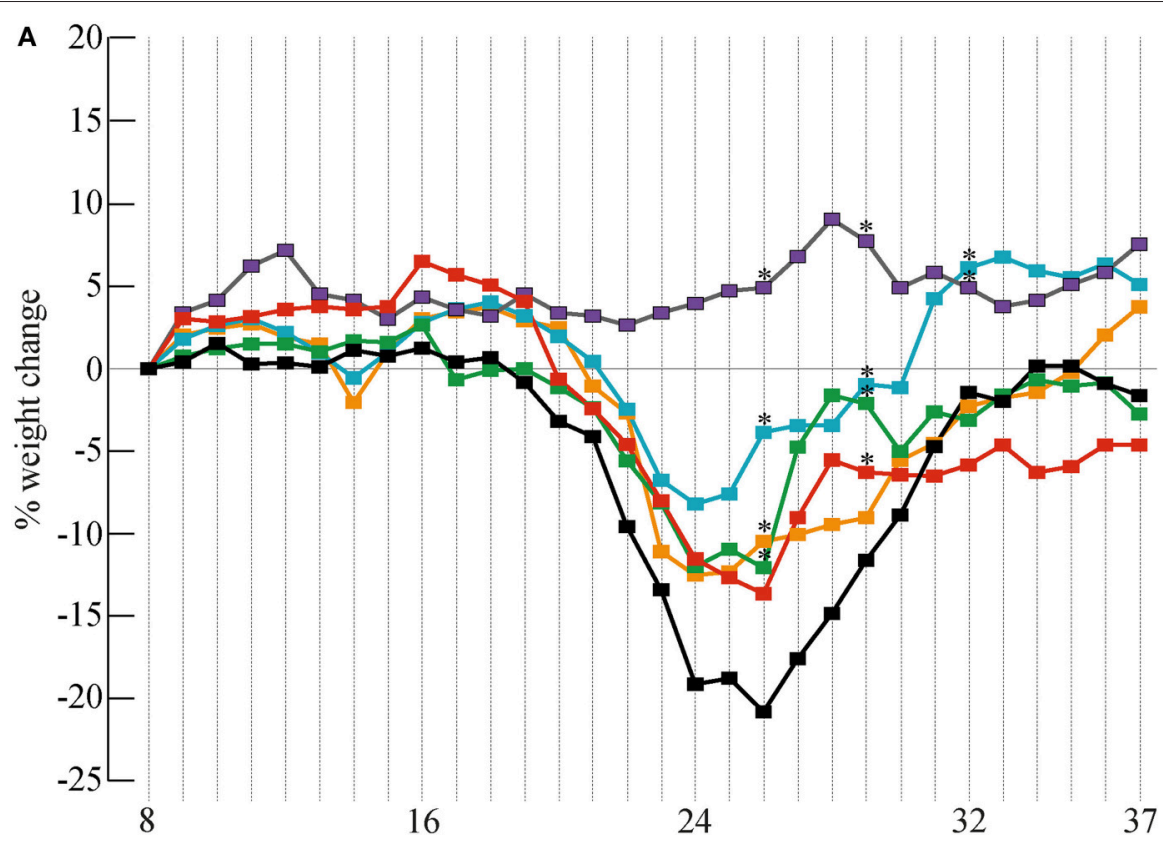

B

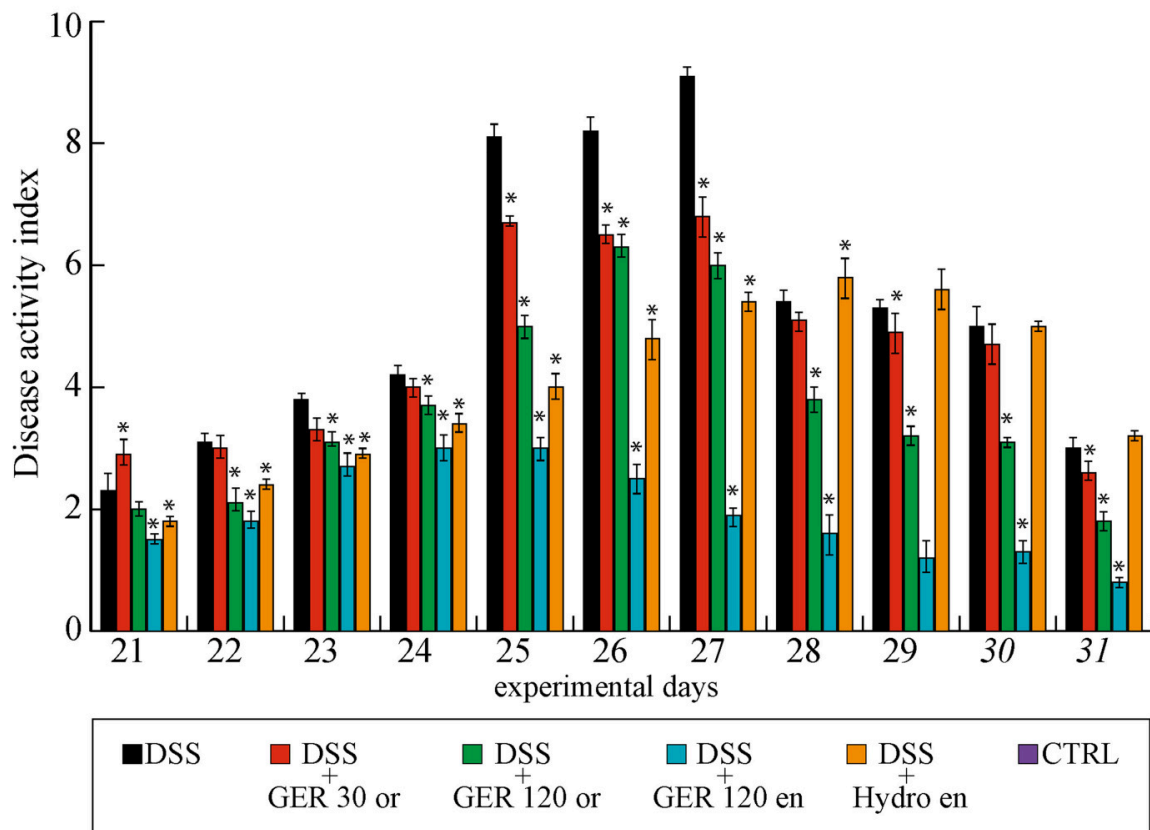

FIGURE 2 | Weight change percentage (A) and disease activity index (DAI) score of colitis (B) in different mice experimental groups. Maximum DAl score was reached between days 25 and 27. Maximum weight loss (22\%) was recorded between days 22 and 27. Weight recovery ends at days 37 . Data are expressed as mean \pm SD. Analysis of variance (one way-ANOVA) was performed (for weight changes only at days 26, 29, and 32) to assess the statistical significance of the differences. ${ }^{*} P<0.05$ if compared to DSS group mean values. Statistical significance for DAl score differences (analysis of variance, one way-ANOVA) are reported in Supplementary Table 1.

even at the dose of $120 \mathrm{mg} \mathrm{kg}^{(-1)}$ (orally administered), did not produced the same marked changes in the microbiota. Indeed, the microbiota composition of mice treated with $\mathrm{Ge}-\mathrm{OH}$
$120 \mathrm{mg} \mathrm{kg}^{(-1)}$ showed a slight increase in Lactobacillaceae, Bacillaceae and Bacteroidetes families (see Supplementary Figure 2). 

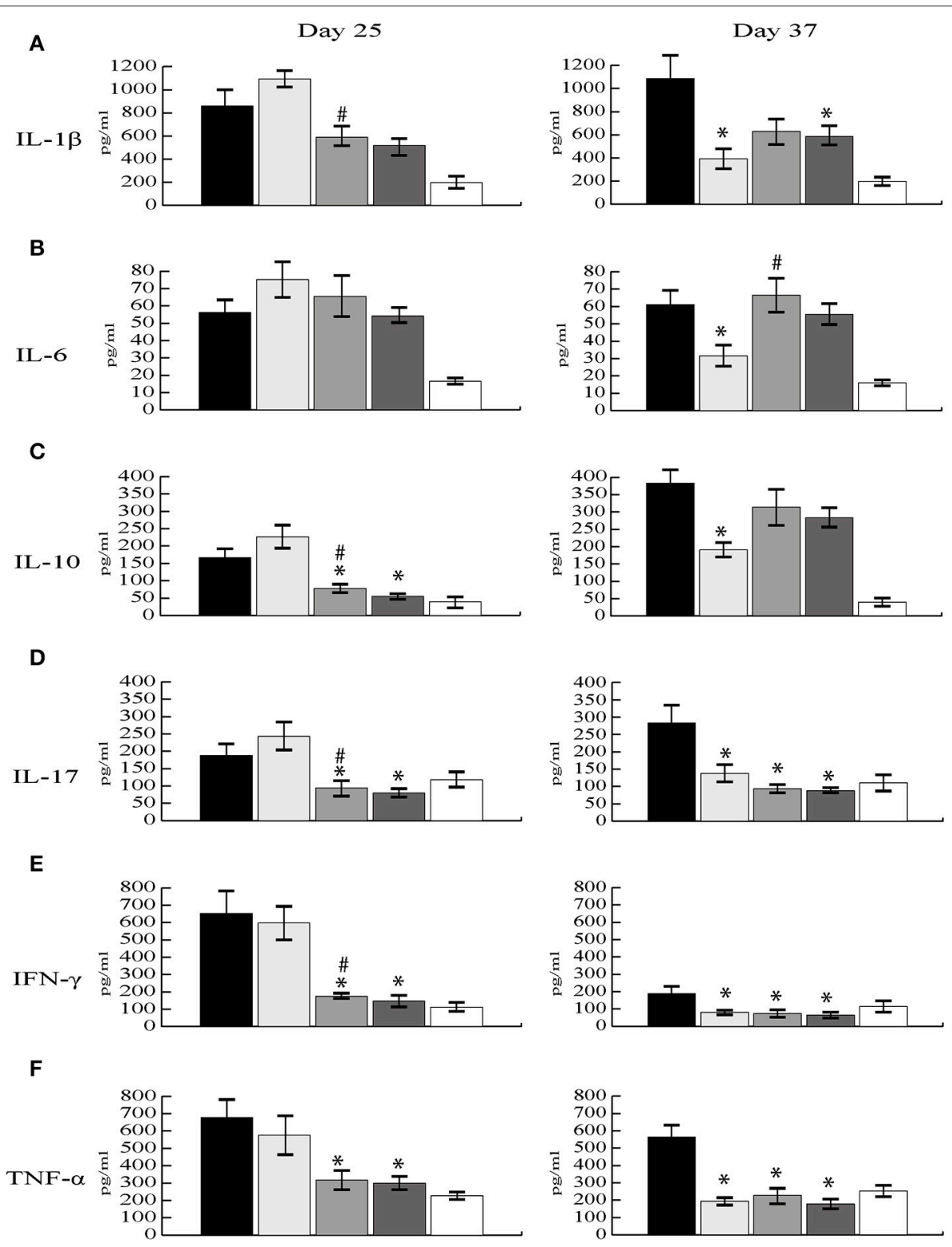

\begin{tabular}{|c|c|c|c|c|}
\hline - DSS & $\square$ CTRL & $\begin{array}{l}\square \text { DSS } \\
\text { GER } 30 \text { or }\end{array}$ & $\begin{array}{l}\square \text { DSS } \\
\text { GER }{ }^{+} 120 \text { or }\end{array}$ & $\begin{array}{c}\text { DSS } \\
\text { GER } 120 \text { en }\end{array}$ \\
\hline
\end{tabular}

FIGURE 3 | Plasma cytokine variations during experimental colitis, measured at days $\mathbf{2 5}$ and $\mathbf{3 7}$. Cytokines were determined using a 6-plex mouse bead immunoassay kit. Levels of IL-1 $\beta$ (A), IL-6 (B), IL-10 (C), IL-17A (D), IFN- $\gamma(\mathbf{E})$, and TNF $\alpha$ (F) are shown. Data are expressed as mean \pm SEM of at least three replicates $(n=9)$. ${ }^{\#} P<0.05$ in the comparison between to $\mathrm{Ge}-\mathrm{OH} 30$ and $\mathrm{Ge}-\mathrm{OH} 120$ or groups. ${ }^{\star} P<0.05$ if compared to DSS group.

\section{Down-Regulation of COX-2 through Ge-OH}

\section{Treatment}

Since COX-2 plays a crucial role in the production of many lipid mediators involved in intestinal inflammation and is one of the major targets of IBD pharmacological therapy, we analyzed COX2 mRNA expression in colon tissues during DSS-induced colitis (Figure 6). Our data support the previously reported finding that COX-2 mRNA significantly increases in the gut wall of DSS-treated mice (De Fazio et al., 2014). At day 25, we observed a significant increase (1.8-fold, $P<0.05)$ in COX-2 expression in the gut wall of DSS-treated mice. $\mathrm{Ge}-\mathrm{OH}$ decrease the COX-2 expression in DSS treated mice returning it to values comparable to those of the control.

\section{DISCUSSION}

Inflammatory bowel disease (IBD) comprises a group of chronic inflammatory conditions affecting the gastrointestinal 


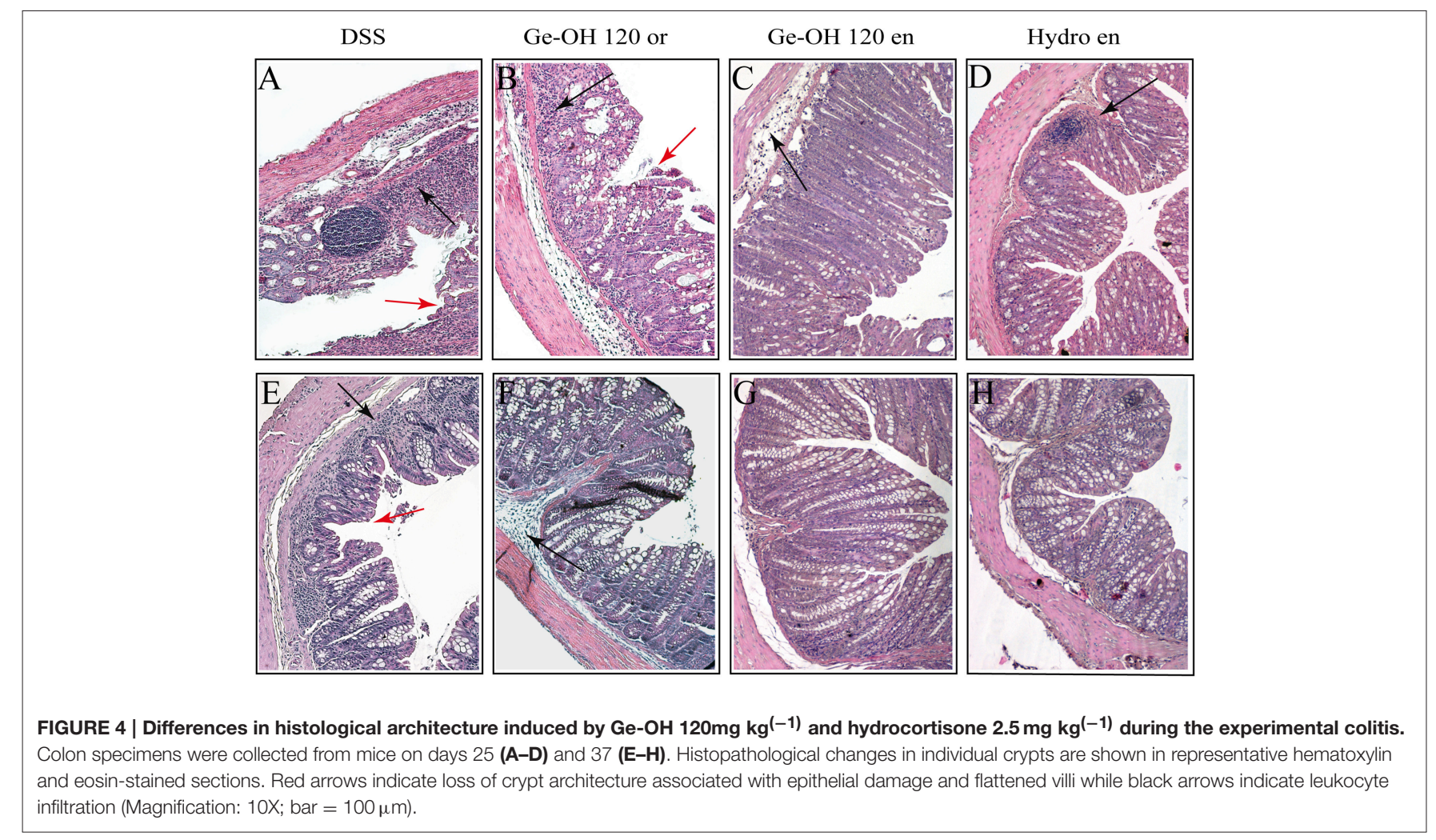

tract. The mucosal immune system of IBD patients has lost the ability to self-regulate and remains chronically activated. IBD is a well-established risk factor for colon cancer (CRC) development, with an increasing incidence linked to younger age at IBD diagnosis, longer IBD duration, and more severe intestinal inflammation. Conventional IBD therapies include COX-2 inhibitors (aminosalicylates and their derivatives), corticosteroids, immunomodulatory drugs, antibiotics, and biologic drugs such as the monoclonal antibody against tumor necrosis factor alpha $(\mathrm{TNF} \alpha)$, a pivotal pro-inflammatory cytokine able to start and maintain the inflammatory process in the gut. Besides antibiotics, probiotics have also been used in the treatment of ulcerative colitis to counteract dysbiosis (Bibiloni et al., 2005). Since, IBD usually relapses, all these therapies require long-term administration.

$\mathrm{Ge}-\mathrm{OH}$ is a non-toxic compound, classified as Generally Recognized As Safe (GRAS) by the US Food and Drug Administration. The European Food Security Agency (EFSA) hazard assessment conclusion for Ge-OH established a Derived No Effect Level (DNEL) of $13.5 \mathrm{mg} \mathrm{kg}^{(-1)}$ for humans (General Population-Hazard via oral route), corresponding to $100-120 \mathrm{mg} \mathrm{kg}^{(-1)}$ in mice. Ge-OH is currently receiving substantial attention for its antitumorigenic, anti-inflammatory, and antimicrobial effects that have been clearly demonstrated in vitro. Nevertheless, its role as an anti-dysbiotic agent in colon inflammation has never been investigated. Our study adopted a mouse model of DSS-induced moderate to severe colitis to evaluate the antimicrobial and anti-inflammatory therapeutic activity of $\mathrm{Ge}-\mathrm{OH}$ doses considered safe.
Ge-OH, orally administered at 30 and $120 \mathrm{mg} \mathrm{kg}^{(-1)}$ halved the mice weight loss and reduced the disease activity index (DAI) of colitis. At histological level, $\mathrm{Ge}-\mathrm{OH}$ was able to preserve crypt architecture and decrease leukocyte infiltration, with a much more evident effect at the higher dose (both enema or orally administered). Moreover, enema-administered Ge-OH strongly improved signs of colitis maintaining a lower DAI and preserving colon mucosa integrity. These clinical observations are further supported by a significant reduction of COX-2 mRNA expression in the colonic mucosa of $\mathrm{Ge}-\mathrm{OH}$-treated mice.

Circulating cytokine levels are indicative of the overall inflammatory status of animals, with IL-1, IL-6, IL-17, and TNF $\alpha$ playing a key role in the pathogenesis of IBD (Muzes et al., 2012). TNF $\alpha$ is a master cytokine in IBD pathogenesis and its orchestrating role in colonic inflammation is confirmed by the efficacy of anti-TNF $\alpha$ therapy in IBD patients (Chaparro et al., 2012). The circulating TNFa level correlates with clinical activity both in ulcerative colitis and Crohn's disease (Bibiloni et al., 2005) and increases in acute phases of DSS colitis (Alex et al., 2009). So, while circulating TNF $\alpha$ and IL-17 levels seem to correlate with the DSS colitis clinical course, IL-1 $\beta$, and IL-10 mainly correlate with the histological damage that tends to become chronic (Alex et al., 2009; De Fazio et al., 2014). The higher oral dose of Ge-OH significantly reduced circulating TNF $\alpha$ and IL17 in Ge-OH-treated mice after weight recovery at the end of the experiments. This decrease was equally evident after $\mathrm{Ge}-\mathrm{OH}$ enema administration. These results are in agreement with those obtained by Medicherla et al. (2015) who found a significantly reduced expression of the major pro-inflammatory cytokines 


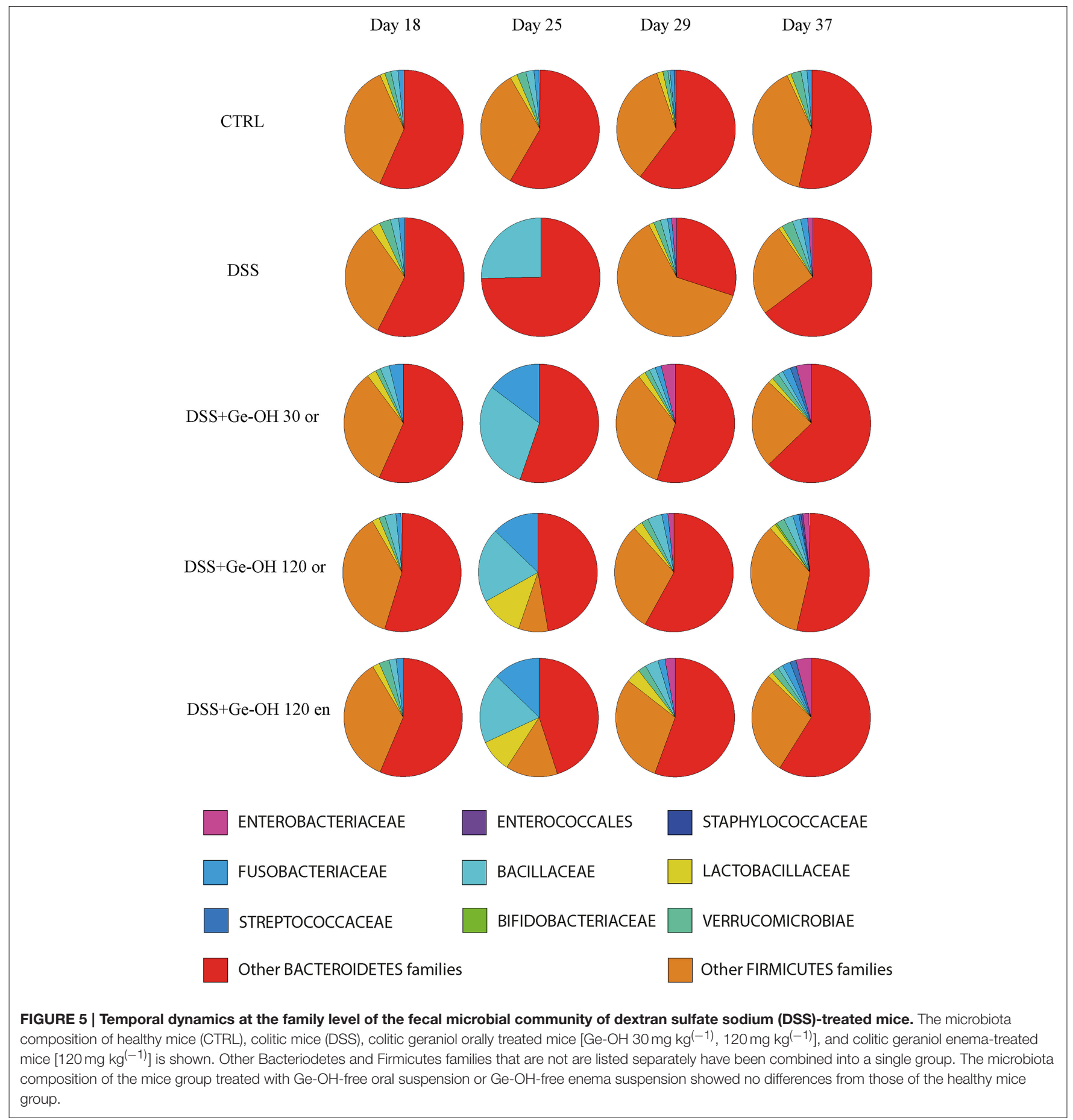

in the colon specimens (TNF- $\alpha$, IL- $1 \beta$, and IL-6), associated with reduced total and nuclear amounts of NF- $\kappa \mathrm{B}(\mathrm{p} 65)$ after oral administration of $\mathrm{Ge}-\mathrm{OH}\left[50\right.$ and $\left.100 \mathrm{mg} \mathrm{Kg}{ }^{(-1)}\right]$. They also identified an antioxidant activity of $\mathrm{Ge}-\mathrm{OH}$ at colon level, evaluated as a decrease in lipid peroxidation marker.

DSS treatment compromises gut microbiota homeostasis, resulting in a dysbiosis characterized by a transient reduction of dominant mutualistic microbiota components such as Bacteroidetes, confirming previous findings (Nagalingam et al.,
2011). Ge-OH oral and enema treatment at $120 \mathrm{mg} \mathrm{kg}^{(-1)}$ protects DSS-treated mice against this transient reduction of Bacteroidetes, boosting a faster recovery of a healthy microbiota profile. Interestingly, $120 \mathrm{mg} \mathrm{kg}^{(-1)}$ geraniol-treated mice presented a transient increase in the relative abundance of Lactobacillaceae from day 25 to day 29. This raises the question of whether the transient $\mathrm{Ge}-\mathrm{OH}$-dependent increase in Lactobacillaceae, heralding the recovery of a healthy profile, is somehow involved in promoting a faster recovery from 


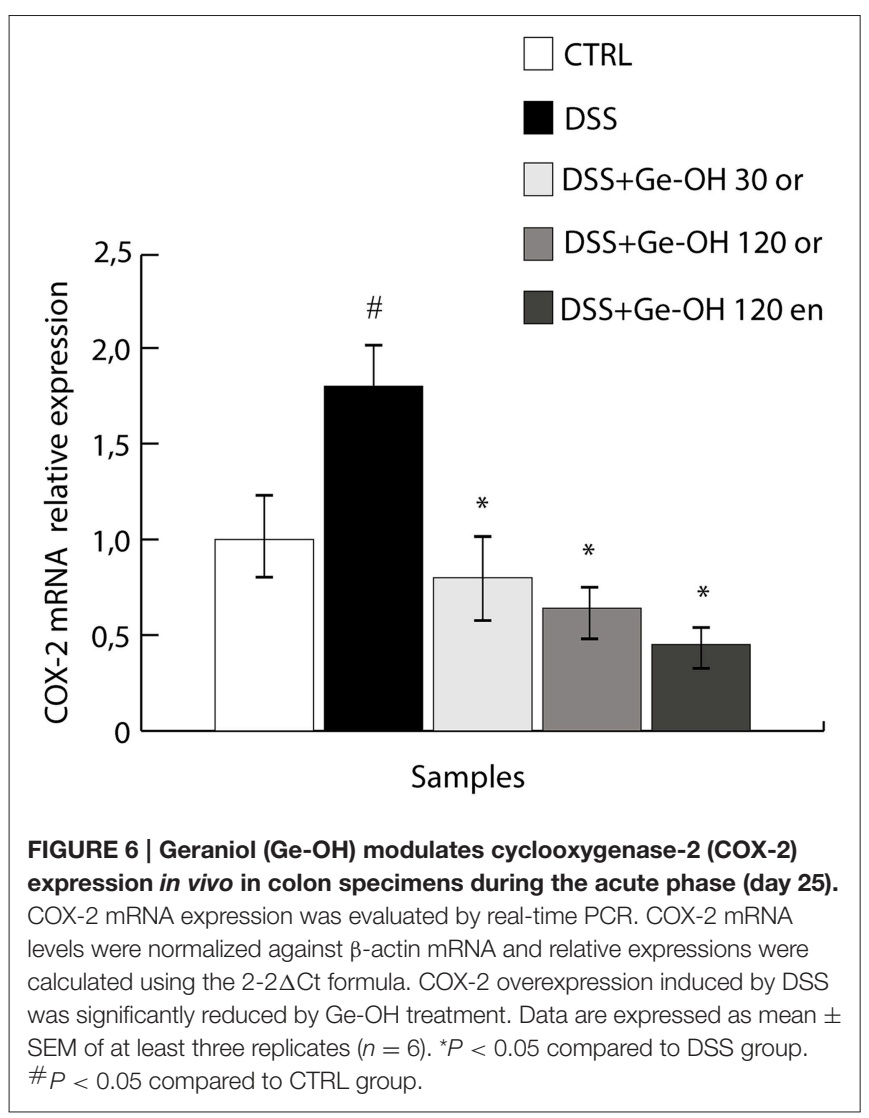

DSS-associated dysbiosis. Dysbiosis always includes a decreased bacterial biodiversity (Honda and Littman, 2012). GeOH $120 \mathrm{mg}$ $\mathrm{kg}^{(-1)}$ treatment was able to increase bacterial biodiversity in DSS-treated mice starting from the 10th day of $\mathrm{Ge}-\mathrm{OH}$ assumption. It is likely that the decreased inflammation we observed in $\mathrm{Ge}-\mathrm{OH}$ oral and enema-treated mice during colitis recovery is also due to the healthy microbiota status found in these mice.

The central finding of this study in a colitis model is the multi-target effect of Ge-OH treatment that simultaneously targeted dysbiosis, local, and systemic inflammation and mucosal damage. The decreased activity of COX-2 in colon specimens is a clear demonstration of the anti-inflammatory effect of $\mathrm{Ge}-\mathrm{OH}$ that contributes to the decreased mucosal damage. This effect certainly involves the colonic mucosa, even if it is reasonable to assume that in vivo Ge-OH may also target $\mathrm{COX}-2$

\section{REFERENCES}

Ahmad, S. T., Arjumand, W., Seth, A., Nafees, S., Rashid, S., Ali, N., et al. (2011). Preclinical renal cancer chemopreventive efficacy of geraniol by modulation of multiple molecular pathways. Toxicology 290, 69-81. doi: 10.1016/j.tox.2011. 08.020

Alex, P., Zachos, N. C., Nguyen, T., Gonzales, L., Chen, T. E., Conklin, L. S., et al. (2009). Centola Distinct cytokine patterns expression in immune system cells inside the colon wall (Su et al., 2010).

\section{CONCLUSIONS}

IBD therapy is based on the use of anti-inflammatory molecules and immunomodulatory agents that act by strongly and nonspecifically inhibiting the inflammatory response but their longterm use might trigger the onset of severe side-effects. The effects of $\mathrm{Ge}-\mathrm{OH}$ could be of great importance in the treatment of human IBD. Since geraniol's antimicrobial effect does not seem to induce bacterial resistance, a phenomenon commonly observed with conventional antibiotic drugs, it would be very interesting to ascertain whether $\mathrm{Ge}-\mathrm{OH}$ is able to control dysbiosis and inflammatory status in human IBD patients. In addition, Ge-OH's anti-tumor activities could help reduce the risk of CRC in IBD patients. Thus, this investigation represents a preclinical assessment prior to developing further studies on the effects of oral and enema $\mathrm{Ge}-\mathrm{OH}$ administration in patients with gut inflammation and/or dysbiosis. Since Ge$\mathrm{OH}$ has its peak therapeutic effect on colitis when directly administered into the colon, it is of great importance to find oral delivery systems able to inhibit intestinal $\mathrm{Ge}-\mathrm{OH}$ absorption after its oral administration. On the contrary, $\mathrm{Ge}-\mathrm{OH}$ without any delivery system may be orally administered to obtain a systemic anti-inflammatory effect or to target other organs, such as brain.

\section{AUTHOR CONTRIBUTIONS}

Participated in research design: LD, MV, MC, FR, and ES. Conducted the experiments: LD, MV, AS, EC, and CR. Performed data analysis: MC, ES, and MC. Wrote the manuscript: LD and ES.

\section{ACKNOWLEDGMENTS}

This study was supported by Xeda international, RD7 Z.A. La crau, 13670 Saint Andiol, France and by University of Bologna (RFO). The authors thank Dr. A. Sardo for his technical and moral support.

\section{SUPPLEMENTARY MATERIAL}

The Supplementary Material for this article can be found online at: http://journal.frontiersin.org/article/10.3389/fphar. 2016.00038

identified from multiplex profiles of murine DSS and TNBSinduced colitis. Inflamm. Bowel Dis. 15, 341-352. doi: 10.1002/ibd. 20753

Bakkali, F., Averbeck, S., Averbeck, D., and Idaomar, M. (2008). Biological effects of essential oils-a review. Food Chem. Toxicol. 46, 446-475. doi: 10.1016/j.fct.2007.09.106

Bibiloni, R., Fedorak, R. N., Tannock, G. W., Madsen, K. L., Gionchetti, P., Campieri, M., et al. (2005). VSL\#3 probiotic-mixture induces remission in 
patients with active ulcerative colitis. Am. J. Gastroenterol. 100, 1539-1546. doi: 10.1111/j.1572-0241.2005.41794.x

Burt, S. (2004). Essential oils: their antibacterial properties and potential applications in foods-a review. Int. J. Food Microbiol. 94, 223-253. doi: 10.1016/j.ijfoodmicro.2004.03.022

Candela, M., Consolandi, C., Severgnini, M., Biagi, E., Castiglioni., B., Vitali, B., et al. (2010). High taxonomic level fingerprint of the human intestinal microbiota by ligase detection reaction-universal array approach. BMC Microbiol. 10:116. doi: 10.1186/1471-2180-10-116

Candela, M., Rampelli, S., Turroni, S., Severgnini, M., Consolandi, C., De Bellis, et al. (2012). Unbalance of intestinal microbiota in atopic children. BMC Microbiol. 12:95. doi: 10.1186/1471-2180-12-95

Castiglioni, B., Rizzi, E., Frosini, A., Sivonen, K., Rajaniemi, P., Rantala, A., et al. (2004). Development of a universal microarray based on the ligation detection reaction and 16S rRNA gene polymorphism to target diversity of cyanobacteria. Appl. Environ. Microbiol. 70, 7161-7172. doi: 10.1128/AEM.70.12.71617172.2004

Chaparro, M., Guerra, I., Muñoz-Linares, P., and Gisbert, J. P. (2012). Systematic review: antibodies and anti-TNF-alpha levels in inflammatory bowel disease. Aliment. Pharmacol. Ther. 35, 971-986. doi: 10.1111/j.1365-2036.2012. 05057.x

Chaudhary, S. C., Siddiqui, M. S., Athar, M., and Alam, M. S. (2013). Geraniol inhibits murine skin tumorigenesis by modulating COX-2 expression, RasERK1/2 signaling pathway and apoptosis. J. Appl. Toxicol. 2013, 828-837. doi: 10.1002/jat.2739

De Fazio, L., Cavazza, E., Spisni, E., Strillacci, A., Centanni, M., Candela, M., et al. (2014). Longitudinal analysis of inflammation and microbiota dynamics in a model of mild chronic dextran sulphate sodium-induced colitis in mice. World J. Gastroenterol. 20, 2051-2061. doi: 10.3748/wjg.v20.i8.2051

Di Pasqua, R., Hoskins, N., Betts, G., and Mauriello, G. (2006).Changes in membrane fatty acids composition of microbial cells induced by addition of thymol, carvacrol, limonene, cinnamaldehyde, and eugenol in the growing media. J. Agric. Food Chem. 54, 2745-2749. doi: 10.1021/jf0 527221

Fang, R., Jiang, C. H., Wang, X. Y., Zhang, H. M., Liu, Z. L., Zhou, L., et al. (2010). Insecticidal activity of essential oil of Carum Carvi fruits from China and its main components against two grain storage insects. Molecules 15, 9391-9402. doi: 10.3390/molecules15129391

Heinlein, A., Metzger, M., Walles, H., and Buettner, A. (2014). Transport of hop aroma compounds across Caco-2 monolayers. Food Funct. 5, 2719-2730. doi: 10.1039/C3FO60675A

Honda, K., and Littman, D. R. (2012). The microbiome in infectious disease and inflammation. Annu. Rev. Immunol. 30, 759-795. doi: 10.1146/annurevimmunol-020711-074937

Kamada, N., Seo, S. U., Chen, G. Y., and Núñez, G. (2013). Role of the gut microbiota in immunity and inflammatory disease. Nat. Rev. Immunol. 13, 321-335. doi: 10.1038/nri3430

Khan, A. Q., Khan, R., Qamar, W., Lateef, A., Rehman, M. U., Tahir, M., et al. (2013). Geraniol attenuates 12-O-tetradecanoylphorbol-13-acetate (TPA)induced oxidative stress and inflammation in mouse skin: possible role of p38 MAP Kinase and NF-кB. Exp. Mol. Pathol. 94, 419-429. doi: 10.1016/j.yexmp.2013.01.006

Koloski, N. A., Bret, L., and Radford-Smith, G. (2008). Hygiene: hypothesis in inflammatory bowel disease: a critical review of the literature. World J. Gastroenterol. 14, 165-173. doi: 10.3748/wjg.14.165

Laukens, D., Brigitta, M., Brinkman, R. J., De Vos, M., and Vandenabeele, P. (2015). Heterogeneity of the gut microbiome in mice: guidelines for optimizing experimental design. FEMS Microbiol. Rev. 40, 117-132. doi: 10.1093/femsre/fuv036

Marcuzzi, A., Crovella, S., and Pontillo, A. (2011). Geraniol rescues inflammation in cellular and animal models of mevalonate kinase deficiency. In Vivo 25, 87-92.

Medicherla, K., Sahu, B. D., Kuncha, M., Kumar, J. M., and Sudhakar, G., Sistla, R. (2015). Oral administration of geraniol ameliorates acute experimental murine colitis by inhibiting pro-inflammatory cytokines and NF-кB signaling. Food Funct. 6, 2984-2995. doi: 10.1039/c5fo00405e
Missaghi, B., Herman, W., Barkema, K. L., and Ghosh, M. S. (2014). Perturbation of the human microbiome as a contributor to inflammatory bowel disease. Pathogens 3, 510-527. doi: 10.3390/pathogens3030510

Muzes, G., Molnár, B., Tulassay, Z., and Sipos, F. (2012). Changes of the cytokine profile in inflammatory bowel diseases. World J. Gastroenterol. 18, 5848-5861. doi: 10.3748/wjg.v18.i41.5848

Nagalingam, N. A., Kao, J. Y., and Young, V. B. (2011). Microbial ecology of the murine gut associated with the development of dextran sodium sulfate-induced colitis. Inflamm. Bowel Dis. 17, 917-926. doi: 10.1002/ibd.21462

Oussalah, M., Caillet, S., Salmiéri, S., Saucier, L., and Lacroix, M. (2007). Antimicrobial effects of alginate-based films containing essential oils on Listeria monocytogenes and Salmonella typhimurium present in bologna and ham. J. Food Prot. 70, 901-908.

Oviedo-Rondón, E. O., Hume, M. E., Hernández, C., and Clemente-Hernández, S. (2006). Intestinal microbial ecology of broilers vaccinated and challenged with mixed Eimeria species, and supplemented with essential oil blends 2006. Poult Sci. 85, 854-860. doi: 10.1093/ps/85.5.854

Rekha, K. R., Selvakumar, G. P., Santha, K., and Inmozhi Sivakamasundari, R. (2013). Geraniol attenuates $\alpha$-synuclein expression and neuromuscular impairment through increase dopamine content in MPTP intoxicated mice by dose dependent manner. Biochem Biophys Res Commun. 440, 664-670. doi: 10.1016/j.bbrc.2013.09.122

Singh, B. K., Tripathi, M., Chaudhari, B. P., Pandey, P. K., and Kakkar, P. (2012). Natural terpenes prevent mitochondrial dysfunction, oxidative stress and release of apoptotic proteins during nimesulide-hepatotoxicity in rats. PLoS ONE 7:e34200. doi: 10.1371/journal.pone.0034200

Solórzano-Santos, F., and Miranda-Novales, M. G. (2012). Essential oils from aromatic herbs as antimicrobial agents. Curr. Opin. Biotechnol. 23, 136-141. doi: 10.1016/j.copbio.2011.08.005

Strillacci, A., Griffoni, C., Lazzarini, G., Valerii, M. C., Di Molfetta, Rizzello, F., et al. (2010). Selective cyclooxygenase-2 silencing mediated by engineered $E$. coli and RNA interference induces anti-tumour effects in human colon cancer cells. $\mathrm{Br}$. J. Cancer 103, 975-986. doi: 10.1038/sj.bjc.6605859

Su, Y. W., Chao, S. H., Lee, M. H., Ou, T. Y., and Tsai, Y. C. (2010). Inhibitory effects of citronellol and geraniol on nitric oxide and prostaglandin E(2)production in macrophages. Planta Med. 76, 1666-1671. doi: 10.1055/s0030-1249947

Thapa, D., Losa, R., Zweifel, B., and Wallace, R. J. (2012). Sensitivity of pathogenic and commensal bacteria from the human colon to essential oils. Microbiology 158, 2870-2877. doi: 10.1099/mic.0.061127-0

Thompson, A., Meah, D., Ahmed, N., Conniff-Jenkins, R., Chileshe, E., Phillips, C. O., et al. (2013). Comparison of the antibacterial activity of essential oils and extracts of medicinal and culinary herbs to investigate potential new treatments for irritable bowel syndrome. BMC Complement. Altern. Med. 13:338. doi: $10.1186 / 1472-6882-13-338$

Turina, A. V., Nolan, M. V., Zygadlo, J. A., and Perillo, M. A. (2006). Natural terpenes: self-assembly and membrane partitioning. Biophys. Chem. 122, 101-113. doi: 10.1016/j.bpc.2006.02.007

Wallace, R. J., Oleszek, W., Franz, C., Hahn, I., Baser, K. H., Mathe, A., et al. (2010). Dietary plant bioactives for poultry health and productivity. Br. Poult. Sci. 51, 461-487. doi: 10.1080/00071668.2010.506908

Yaung, S. J., Church, G. M., and Wang, H. H. (2014). Recent progress in engineering human-associated microbiomes. Methods Mol. Biol. 1151, 3-25. doi: 10.1007/978-1-4939-0554-6_1

Conflict of Interest Statement: The authors declare that the research was conducted in the absence of any commercial or financial relationships that could be construed as a potential conflict of interest.

Copyright (C) 2016 De Fazio, Spisni, Cavazza, Strillacci, Candela, Centanni, Ricci, Rizzello, Campieri and Valerii. This is an open-access article distributed under the terms of the Creative Commons Attribution License (CC BY). The use, distribution or reproduction in other forums is permitted, provided the original author(s) or licensor are credited and that the original publication in this journal is cited, in accordance with accepted academic practice. No use, distribution or reproduction is permitted which does not comply with these terms. 